\title{
FIXED POINT AND COINCIDENCE POINT THEOREMS
}

\author{
ARJAMAND BANO AND SAIMA NAHEED
}

\begin{abstract}
In this paper, we present a generalization of some fixed point and coincidence point theorems using the notion of a $w$-distance on a complete metric space. Consequently, we improve and generalize various results existing in the literature.
\end{abstract}

\section{Introduction and Preliminaries}

In 1969, Nadler [4] extended the well known Banach contraction principle [1], which asserts that "each single-valued contraction self map on a complete metric space has a unique fixed point.", to multi-valued mapping using the concept of Hausdorff metric. In [3], Kada et al. introduced a notion of $w$-distance on a metric space and improved several results replacing the involved metric by a generalized distance. In this paper, using the concept of a $w$-distance, we give a generalization of Nadler's fixed point theorem and coincidence point results in complete metric space.

Let $(E, d)$ be a complete metric space and let $C B(E)$ be the collection of all nonempty closed bounded subsets of $E$. For $A, B \in C B(E)$ and $p \in E$, define

$$
D(p, A)=\inf \{d(p, a): a \in A\}
$$

and

$$
H(A, B)=\max \left\{\sup _{a \in A} D(a, B), \sup _{b \in B} D(b, A)\right\}
$$

where $H$ is the Hausdorff metric induced by $d$.

One can easly prove that $(C B(E), H)$ is a complete metric space, whenever $(E, d)$ is a complete metric space ( see e.g. Lemma 8.1.4, of [8]).

Definition 1. An element $p \in E$ is said to be a fixed point of a multi-valued mapping $T: E \rightarrow$ $C B(E)$, if $p \in T(p)$.

Definition 2. Let $C(f, T)$ be the set of coincidence points of $f$ and $T$

i.e. $C(f, T)=\{p \in E: f p \in T p\}$.

Corresponding author: Saima Naheed.

2010 Mathematics Subject Classification. 47H09, 54H25.

Key words and phrases. $w$-distance, Nadler's fixed point theorem, coincidence point, Hausdorff metric. 
Definition 3. A map $\varphi: E \rightarrow R$ is called lower semi-continuous if for any sequence $\left\{p_{n}\right\} \subset E$ with $p_{n} \rightarrow p \in E$ implies $\varphi(p) \leq \liminf _{n \rightarrow \infty} \varphi\left(p_{n}\right)$.

Definition 4. A function $w: E \times E \rightarrow[0, \infty)$ is called a $w$-distance on $E$ if it satisfies the following for any $p_{1}, p_{2}, p_{3} \in E$ such that

1. $w\left(p_{1}, p_{3}\right) \leq w\left(p_{1}, p_{2}\right)+w\left(p_{2}, p_{3}\right)$;

2. a map $w(p,):. E \rightarrow[0, \infty)$ is lower semi-continuous:

3. for any $\varepsilon>0$, there exists $\delta>0$ such that $w\left(p_{3}, p_{1}\right) \leq \delta$ and $w\left(p_{3}, p_{2}\right) \leq \delta$ imply $d\left(p_{1}, p_{2}\right) \leq$ $\varepsilon$.

The metric $d$ is a $w$-distance on $E$. Note that, in general for $p_{1}, p_{2} \in E, w\left(p_{1}, p_{2}\right) \neq w\left(p_{2}, p_{1}\right)$. Define $w(p, A)=\inf \{w(p, a): a \in A\}$ and

$$
H_{w}(A, B)=\max \left\{\sup _{a \in A} w(a, B), \sup _{b \in B} w(b, A)\right\}
$$

It is easy to see that $H_{w}$ is a metric on $C B(E) . H_{w}$ is called the Hausdorff metric induced by $w$.

Lemma 1. Let $E$ be a metric space and $A, B \in C B(E)$. Then for each $a \in A$ and $\varepsilon>0$ there exists an $b \in B$ such that

$$
w(a, b)=H_{w}(A, B)+\varepsilon .
$$

\section{Main Results}

Theorem 1. Let $(E, d)$ be a complete metric space and let $T: E \rightarrow C B(E)$ be a mapping such that $H_{w}\left(T p_{1}, T p_{2}\right) \leq \alpha w\left(p_{1}, p_{2}\right)+\beta\left[w\left(p_{1}, T p_{1}\right)+w\left(p_{2}, T p_{2}\right)\right]+\gamma\left[w\left(p_{1}, T p_{2}\right)+w\left(p_{2}, T p_{1}\right)\right]$ for all $p_{1}, p_{2} \in E$, where $\alpha, \beta, \gamma \geq 0$ and $\alpha+2 \beta+2 \gamma<1$. Then $T$ has a fixed point.

Proof. Let $p_{0} \in E, p_{1} \in T p_{0}$ and define $h=\frac{\alpha+\beta+\gamma}{1-(\beta+\gamma)}$. If $h=0$ then proof is trivial.

Now, assume that $h>0$. Then it follows from Lemma 1 that

$$
\begin{array}{rlrl}
\exists p_{2} \in T p_{1} ; & w\left(p_{1}, p_{2}\right) & \leq H_{w}\left(T p_{0}, T p_{1}\right)+h \\
\exists p_{3} \in T p_{2} ; & w\left(p_{2}, p_{3}\right) & \leq H_{w}\left(T p_{1}, T p_{2}\right)+h^{2}, \\
& \vdots \\
\exists p_{n+1} \in T p_{n} ; & w\left(p_{n}, p_{n+1}\right) & \leq H_{w}\left(T p_{n-1}, T p_{n}\right)+h^{n} .
\end{array}
$$


Hence, we have

$$
\begin{aligned}
w\left(p_{n}, p_{n+1}\right) \leq & H_{w}\left(T p_{n-1}, T p_{n}\right)+h^{n} \\
\leq & \alpha w\left(p_{n-1}, p_{n}\right)+\beta\left[w\left(p_{n}, T p_{n}\right)+w\left(p_{n-1}, T p_{n-1}\right)\right] \\
& +\gamma\left[w\left(p_{n}, T p_{n-1}\right)+w\left(p_{n-1}, T p_{n}\right)\right]+h^{n} \\
\leq & \alpha w\left(p_{n-1}, p_{n}\right)+\beta\left[w\left(p_{n}, p_{n+1}\right)+w\left(p_{n-1}, p_{n}\right)\right] \\
& +\gamma\left[w\left(p_{n-1}, p_{n}\right)+w\left(p_{n}, p_{n+1}\right)\right]+h^{n}
\end{aligned}
$$

for all $n \in \mathrm{N}$. It follows that

$$
w\left(p_{n}, p_{n+1}\right) \leq h w\left(p_{n-1}, p_{n}\right)+\frac{h^{n}}{1-(\beta+\gamma)}
$$

for all $n \in \mathrm{N}$. It can be conclude that

$$
w\left(p_{n}, p_{n+1}\right) \leq h^{n} w\left(p_{0}, p_{1}\right)+\frac{n h^{n}}{1-(\beta+\gamma)}
$$

for all $n \in \mathrm{N}$. Now, since $h<1$, then $\sum_{n=1}^{\infty} w\left(p_{n}, p_{n+1}\right)<\infty$. It follows that $\left\{p_{n}\right\}$ is a Cauchy sequence in $E$. By completeness of $E$, there exists $p^{*} \in E$ such that $\lim _{n \rightarrow \infty} p_{n}=p^{*}$. Now we shall show that $p^{*}$ is a fixed point of $T$. We have

$$
\begin{aligned}
w\left(p^{*}, T p^{*}\right) \leq & w\left(p^{*}, p_{n+1}\right)+w\left(p_{n+1}, T p^{*}\right) \leq w\left(p^{*}, p_{n+1}\right)+H_{w}\left(T p_{n}, T p^{*}\right) \\
\leq & w\left(p^{*}, p_{n+1}\right)+\alpha w\left(p_{n}, p^{*}\right)+\beta\left[w\left(p_{n}, T p_{n}\right)+w\left(p^{*}, T p^{*}\right)\right] \\
& +\gamma\left[w\left(p_{n}, T p^{*}\right)+w\left(p^{*}, T p_{n}\right)\right]
\end{aligned}
$$

for all $n \in \mathrm{N}$. Therefore,

$$
\begin{aligned}
w\left(p^{*}, T p^{*}\right) \leq & w\left(p^{*}, p_{n+1}\right)+\alpha w\left(p_{n}, p^{*}\right)+\beta\left[w\left(p_{n}, p_{n+1}\right)+w\left(p^{*}, T p^{*}\right)\right] \\
& +\gamma\left[w\left(p_{n}, T p^{*}\right)+w\left(p_{n+1}, p^{*}\right)\right]
\end{aligned}
$$

for all $n \in \mathrm{N}$. As $n \rightarrow \infty$, we have

$$
w\left(p^{*}, T p^{*}\right) \leq(\beta+\gamma) w\left(p^{*}, T p^{*}\right)
$$

Since $\beta+\gamma<1$, then $w\left(p^{*}, T p^{*}\right)=0$. It follows that $p^{*} \in T p^{*}$.

Theorem 2. Let $(E, d)$ be a complete metric space and let $T: E \rightarrow C B(E)$ and $f: E \rightarrow E, f$ is continuous mapping which commute with $T$ and $T(E) \subseteq f(E)$. Suppose there exists $h \in(0,1)$ such that

$w\left(T p_{1}, T p_{2}\right) \leq \alpha w\left(f p_{1}, f p_{2}\right)+\beta\left[w\left(f p_{1}, T p_{1}\right)+w\left(f p_{2}, T p_{2}\right)\right]+\gamma\left[w\left(f p_{1}, T p_{2}\right)+w\left(f p_{2}, T p_{1}\right)\right]$ for all $p_{1}, p_{2} \in E$, where $\alpha, \beta, \gamma \geq 0$ and $\alpha+2 \beta+2 \gamma<1$. Then fand Thave a coincidence point. 
Proof. Let $p_{0}$ be an arbitrary but fixed element of $E$ and let $p_{1} \in E$ be a point such that $f p_{1} \in$ $T p_{0}$. Using the definition of the Hausdorff metric and the fact that $f(E) \subseteq T(E)$, we may choose $p_{2} \in E$ such that $f p_{2} \in T p_{1}$ and

$$
w\left(f p_{2}, f p_{1}\right) \leq H_{w}\left(T p_{1}, T p_{0}\right)+h^{2} .
$$

Inductively after $p_{n}$ has been determined, we choose $p_{n+1} \in E$, so that $f p_{n+1} \in T p_{n}$ and

$$
w\left(f p_{n}, f p_{n+1}\right) \leq H_{w}\left(T p_{n-1}, T p_{n}\right)+h^{n}
$$

Then

$$
\begin{aligned}
w\left(f p_{n}, f p_{n+1}\right) \leq & H_{w}\left(T p_{n-1}, T p_{n}\right)+h^{n} \\
\leq & \alpha w\left(f p_{n-1}, f p_{n}\right)+\beta\left[w\left(f p_{n}, T p_{n}\right)+w\left(f p_{n-1}, T p_{n-1}\right)\right] \\
& +\gamma\left[w\left(f p_{n}, T p_{n-1}\right)+w\left(f p_{n-1}, T p_{n}\right)\right]+h^{n} \\
\leq & \alpha w\left(f p_{n-1}, f p_{n}\right)+\beta\left[w\left(f p_{n}, f p_{n+1}\right)+w\left(f p_{n-1}, f p_{n}\right)\right] \\
& +\gamma\left[w\left(f p_{n}, f p_{n+1}\right)+w\left(f p_{n-1}, f p_{n}\right)\right]+h^{n}
\end{aligned}
$$

for all $n \in \mathrm{N}$. It follows that

$$
w\left(f p_{n}, f p_{n+1}\right) \leq h w\left(f p_{n-1}, f p_{n}\right)+\frac{h^{n}}{1-(\beta+\gamma)}
$$

for all $n \in \mathrm{N}$. It can be conclude that

$$
w\left(f p_{n}, f p_{n+1}\right) \leq h^{n} w\left(f p_{0}, f p_{1}\right)+\frac{n h^{n}}{1-(\beta+\gamma)}
$$

for all $n \in \mathrm{N}$. Now , since $h<1$, then $\sum_{n=1}^{\infty} w\left(f p_{n}, f p_{n+1}\right)<\infty$. It follows that $\left\{f p_{n}\right\}$ is a Cauchy sequence in $E$. Since $E$ is complete metric space, there exists a $u \in E$ and $f u=u$. By definition 4 we have

$$
\begin{aligned}
w(T u, f u) \leq & w\left(T u, f p_{n+1}\right)+w\left(f p_{n+1}, f u\right) \\
\leq & H_{w}\left(T u, T p_{n}\right)+w\left(f p_{n+1}, f u\right) \\
\leq & \alpha w\left(f u, f p_{n}\right)+\beta\left[w(f u, T u)+w\left(f p_{n}, T p_{n}\right)\right] \\
& +\gamma\left[w\left(f u, T p_{n}\right)+w\left(f p_{n}, T u\right)\right]+w\left(f p_{n+1}, f u\right) .
\end{aligned}
$$

for all $n \in \mathrm{N}$. Therefore,

$$
\begin{aligned}
w(T u, f u) \leq & \alpha w\left(f u, f p_{n}\right)+\beta\left[w(f u, T u)+w\left(f p_{n}, f p_{n+1}\right)\right] \\
& +\gamma\left[w\left(f u, f p_{n+1}\right)+w\left(f p_{n}, T u\right)\right]+w\left(f p_{n+1}, f u\right)
\end{aligned}
$$

For all $n \in \mathrm{N}$. Taking the limit as $n \rightarrow \infty$, we have

$$
w(T u, f u) \leq(\beta+\gamma) w(f u, T u) .
$$

Since $\beta+\gamma<1$, then $w(T u, f u)=0$. It follows that $f u \in T u$. 
Corollary 1 ([2]). Let $E$ be complete metric space and let $T: E \rightarrow E$ such that

$$
w\left(T p_{1}, T p_{2}\right) \leq \alpha w\left(p_{1}, p_{2}\right)+\beta\left[w\left(p_{1}, T p_{1}\right)+w\left(p_{2}, T p_{2}\right)\right]+\gamma\left[w\left(p_{1}, T p_{2}\right)+w\left(p_{2}, T p_{1}\right)\right]
$$

for all $p_{1}, p_{2} \in E$ where $\alpha, \beta, \gamma \geq 0$ and $\alpha+2 \beta+\gamma<1$. Then $T$ has a fixed point.

Corollary 2. Let E be complete metric space and let $T: E \rightarrow C B(E)$ satisfies

$$
H_{w}\left(T p_{1}, T p_{2}\right) \leq \alpha w\left(p_{1}, p_{2}\right)
$$

for all $p_{1}, p_{2} \in E$, where $0 \leq \alpha<1$. Then $T$ has a fixed point.

Corollary $3([5,6,7])$. Let $E$ be a complete metric space and let $T: E \rightarrow C B(E)$ satisfy

$$
H_{w}\left(T p_{1}, T p_{2}\right) \leq \beta\left[w\left(p_{1}, T p_{1}\right)+w\left(p_{2}, T p_{2}\right)\right]
$$

for all $p_{1}, p_{2} \in E$, where $\beta \in\left[0, \frac{1}{2}\right)$. Then $T$ has a fixed point.

Corollary 4. Let $E$ be complete metric space and let $T: E \rightarrow C B(E)$ satisfy

$$
H_{w}\left(T p_{1}, T p_{2}\right) \leq \gamma\left[w\left(p_{1}, T p_{2}\right)+w\left(p_{2}, T p_{1}\right)\right]
$$

for all $p_{1}, p_{2} \in E$, where $\gamma \in\left[0, \frac{1}{2}\right)$. Then $T$ has a fixed point.

Corollary 5. Let $E$ be complete metric space and let $T: E \rightarrow C B(E)$ satisfy

$$
H_{w}\left(T p_{1}, T p_{2}\right) \leq \alpha w\left(p_{1}, p_{2}\right)+\beta\left[w\left(p_{1}, T p_{1}\right)+w\left(p_{2}, T p_{2}\right)\right]
$$

for all $p_{1}, p_{2} \in E$, where $\alpha+2 \beta<1$. Then $T$ has a fixed point.

Corollary 6. Let $E$ be complete metric space and let $T: E \rightarrow C B(E)$ satisfy

$$
H_{w}\left(T p_{1}, T p_{2}\right) \leq a_{1} w\left(p_{1}, p_{2}\right)+a_{2} w\left(p_{1}, T p_{1}\right)+a_{3} w\left(p_{2}, T p_{2}\right)+a_{4} w\left(p_{1}, T p_{2}\right)+a_{5} w\left(p_{2}, T p_{1}\right)
$$

for all $p_{1}, p_{2} \in E$, where $a_{i} \geq 0$ for each $i \in\{1,2, \ldots, 5\}$ and $\sum_{i=1}^{5} a_{i}<1$. Then $T$ has a fixed point.

\section{References}

[1] S. Banach, Sure operations dans les ensembles abstraits et leur application aux equations integrals, Fund. Math., 3 (1922), 133-181.

[2] G. E. Hardy and T. D. Rogers, A generalization of a fixed point theorem of Reich, Canad. Math. Bull., 16 (1973), 201-206.

[3] O. Kada, T. Suzuki and W. Takahashi, Non convex minimization theorems and fixed point theorems in complete metric space, Math. Japon., 44, (1996), 381-391.

[4] N. B. Nadler J., Multi-valued contraction mappings, Pacific J. Math., 30 (1969), 475-488. 
[5] R. Morales, Generalization of Rakotch's fixed point theorem, Revista de matematica: teoreay aplicaciones. Enero 2002. 9, 25-33.

[6] S. Reich, Fixed points of contractive functions, Boll. Un. Mat. Ital., 5 (1972), 26-42.

[7] S. Reich, Kannan's fixed point theorem, Boll. Un. Mat. Ital., 4 (1971), 1-11.

[8] I. A. Rus, Generalized contraction and Applications, Cluj University Press, Cluj-Nappa, 2001.

Department of Mathematics, Gomal University Dera Ismail Khan, Pakistan.

E-mail: arjamandbano2002@yahoo.com

E-mail: saima_naheed86@yahoo.com 